\title{
Synthesis of New 3, 5-Diaryl-4, 5-Dihydroisoxazole-4- Carbonitriles via 1, 3-Dipolar Cycloaddition Reaction
}

\author{
P. Jayaroopa, G. Vasanth Kumar, N. Renuka and K. Ajay Kumar* \\ Department of Chemistry, Yuvaraja's College, University of Mysore, Mysore, India.
}

\begin{abstract}
Aromatic aldoximes 1a-i undergo oxidative dehydrogenation with $\mathrm{CrO}_{2}$ to give nitrile oxides, which are trapped in situ by 4-methoxycinnamonitrile 2 to afford of ethyl 3,5-diaryl-4,5-dihydroisoxazole-4carbonitriles $3 a-i$ in good yield.
\end{abstract}

Key words: Isoxazoles, isoxazolines, magtreive ${ }^{T M}$, antimicrobial, antioxidant.

\section{Introduction}

Isoxazoles and isoxazolines are very useful heterocycles in organic and heterocyclic chemistry [1]. Isoxazolines also serves as important building blocks for the synthesis of various biologically active molecules [2]. The isoxazoles are known to exhibit significant number of biological applications such as hypoglycemic, analgesic, anti-inflammatory and HIV-inhibitory activity [3], also found to exhibit antibacterial [4], antifungal [5], antioxidant [6], potent selective agonists at human cloned dopamine D4 receptors [7], COX-2 inhibitory [8], antinociceptive [9], anticancer [10], and antibiotic, antitumour, insecticidal activities [1,2]. They serve as prodrug for the anti-arithretic agent [11]. Vijay V. Dabholkar and co-workers [12] reported the synthesis and their antimicrobial activity of fused isoxazolines. Joshi and et al [13] reported that isoxazoles acts as potential antitubercular agents.

The most convenient synthesis of isoxazoline and isoxazole ring system has been executed in the literature via 1,3-dipolar cycloaddition reactions of alkenes and alkynes with nitrile oxides generated in situ from aldoximes [2,14]. Rai et al developed new methods for generating nitrile oxides involving oxidative dehydrogenation of aldoximes using oxidants such as chloramine- $\mathrm{T}$ and mercuric acetate [15]. Treatment of aldoximes with Magtreive $\left(\mathrm{CrO}_{2}\right)$ in presence of dipolarophile furnished a variety of isoxazolines and isoxazoles as 1,3-dipoar cycloaddition products [16,17]. It was reported that nitrile oxides preferentially adds to olefinic $\mathrm{C}=\mathrm{C}$ bond rather than $\mathrm{C}-\mathrm{N}$ triple bond of acrylonitrile to form isoxazolines [18]. The classical method employed for the synthesis of isoxazolines involves 1,3-dipolar cycloaddition reactions of nitrile oxides to alkenes [19,20]. This paper describes the successful synthesis of new 3,5-diaryl-4,5-dihydroisoxazole-4-carbonitriles via 1.3dipolar cycloaddition reactions

\section{Materials And Methods}

The chemicals/reagents used were purchased from sigma-aldrich chemicals (India) and Merck Chemicals (India). IR spectra were recorded on a Nujol mull on Shimadzu 8300 spectrometer. The ${ }^{1} \mathrm{H}$ NMR and ${ }^{13} \mathrm{C}$ NMR spectra were recorded on a Bruker supercon $400 \mathrm{MHz}$ spectrophotometer using $\mathrm{CDCl}_{3}$ as solvent and TMS as an internal standard. The Chemical shifts are expressed in $\delta \mathrm{ppm}$. Mass spectra were obtained on Shimadzu LCMS-2010A spectrophotometer (chemical ionization) and the important fragments are given with the relative intensities in the brackets. Elemental analysis was obtained on a Thermo Finnigan Flash EA 1112 CHN analyser. Thin layer chromatography (TLC) was performed on a pre-coated Silica Gel sheets (HF 254, sd-fine) using benzene: ethyl acetate (7:2) eluent and visualization of the spots was done in iodine vapour and UV light. Chromatographic separations were carried out on silica gel (70-230 mesh, Merck) column using hexane: ethyl acetate (8:1) as eluent.

In a general 1,3-dipolar cycloaddition reaction, a mixture of aromatic aldoximes 2, 4-methoxy cinnamonitrile 1, and $\mathrm{CrO}_{2}$ in acetonitrile was stirred at $80^{\circ} \mathrm{C}$ for 2 hours. The progress of the reaction was monitored by TLC. After the completion of the reaction and usual work up, the reaction mixture gave one major spot corresponding to the product 3,5-diaryl-4,5-dihydroisoxazole-4-carbonitrile 3 in TLC, and two minor spots corresponding to the un-reacted precursors. The products $\mathbf{3}$ were separated by column chromatography using hexane:ethyl acetate $(8: 1 \mathrm{v} / \mathrm{v})$ and are obtained in $52-62 \%$ yield (Scheme-1). 


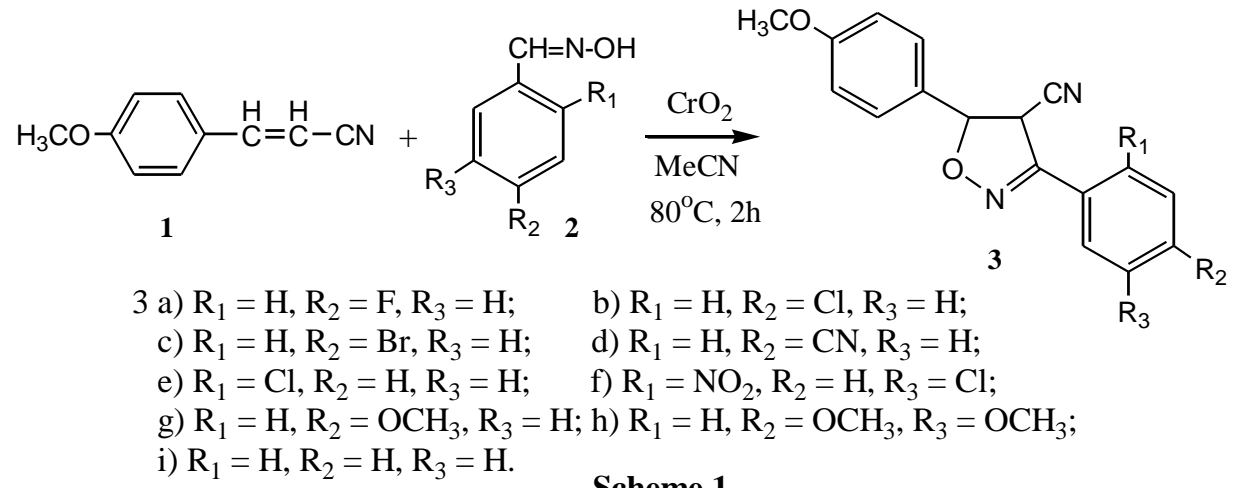

\section{Scheme 1}

\section{Results And Discussion}

The general synthetic pathway employed is depicted in the scheme-1. The structures of the cycloadducts were provided by IR, ${ }^{1} \mathrm{H}$ NMR, ${ }^{13} \mathrm{C}$ NMR, MS studies and elemental analysis. For instance, in IR spectra, the cycloadducts 3 gave the absorptions bands in the region 1650-1675 $\mathrm{cm}^{-1}$ for $\mathrm{C}=\mathrm{N}$ (str) group which is a clear indication of the formation of cycloadducts, a strong and sharp absorption bands in the region $2220-2240 \mathrm{~cm}^{-1}$ for $\mathrm{CN}$ (str) which supports the fact that the $\mathrm{C}-\mathrm{N}$ triple bond of $\mathrm{CN}$ group is unaffected during the cycloaddition reaction.

In ${ }^{1} \mathrm{H}$ NMR spectra, all substituted-4,5-dihydroisoxazole-4-carbonitriles 3 showed the peaks due to aromatic and substituent protons at the expected region. The consistent pattern signals due to $\mathrm{C}_{4}-\mathrm{H}$ appear as doublet in the region $\delta 5.00-5.29 \mathrm{ppm}$., while signals due to $\mathrm{C}_{5}-\mathrm{H}$ appears as doublet in the region $\delta 5.50-5.71$ ppm. The coupling constant $(J)$ values calculated for $\mathrm{C}_{4}-\mathrm{H}$ and $\mathrm{C}_{5}-\mathrm{H}$ were in range 7.0-9.6 $\mathrm{Hz}$, these values suggests that both $\mathrm{C}_{4}-\mathrm{H}$ and $\mathrm{C}_{5}-\mathrm{H}$ are cis orientation and the cycloaddition took place in cis fashion. The appearance of these proton signals in the downfield was expected due to the strong electron withdrawing $-\mathrm{CN}$ group and aromatic ring bonded to $\mathrm{C}_{4^{-}}$and $\mathrm{C}_{5^{-}}$atoms respectively, which favors the formation of cycloadducts. In ${ }^{13} \mathrm{C}$ NMR, all products gave the signals due to aromatic and substituent carbons at the expected region. The signals due to newly formed $\mathrm{C}_{4}$-carbon appeared in the region $\delta_{\mathrm{c}}$ 21.2-23.6 ppm, while, $\mathrm{C}_{5}$-carbon showed the signals in the region $\delta_{\mathrm{c}} 66.59-66.94 \mathrm{ppm}$ and $\mathrm{C}_{3}$-carbon showed the signals in the region $\delta_{\mathrm{c}}$ 161.3-164.7 ppm. The signals due to $\mathrm{CN}$ group carbon appear in the region $\delta_{\mathrm{c}} 116.2-118.0 \mathrm{ppm}$., which shows that the $\mathrm{CN}$ triple bond is unaffected during cycloaddition and is retained in the product. All substituted-4,5-dihydroisoxazole-4carbonitriles 3 gave significantly stable molecular ion peaks with a relative abundance ranging from $08-46 \%$ and base peak at $\left(\mathrm{MH}^{+}\right)$. Further, all showed satisfactorily CHN analysis with a deviation of $\pm 0.10 \%$ from the theoretically calculated values. All these observations strongly favor the formation of the cycloadducts.

\section{Experimental}

4.1 Typical procedure for the preparation of 3-(4-fluorophenyl)-5-(4-methoxyphenyl)-4,5-dihydroisoxazole4-carbonitrile 3a: A mixture of 4-fluorobenzaldehyde oxime 2 (106mg, $0.76 \mathrm{mmol}$, 1.2equiv), 3-(4methoxyphenyl) acrylonitrile $\mathbf{1}$ (100mg, $0.63 \mathrm{mmol}, 1.0$ equiv) were dissolved in $3 \mathrm{ml}$ acetonitrile. Magtrieve $\mathrm{e}^{\mathrm{TM}}$ (530mg, $6.31 \mathrm{mmol}, 10$ equiv) was added and the reaction mixture was stirred under heating at $80^{\circ} \mathrm{C}$ for $2 \mathrm{~h}$. The progress of the reaction was monitored by TLC. After completion of the reaction, the reaction mixture was filtered through Celite bed. Magtrieve was washed with ethyl acetate $(20 \mathrm{ml} \times 2)$. The combined filtrate was condensed to give the crude product, which was purified by column chromatography using hexane:ethyl acetate $(8: 1 \mathrm{v} / \mathrm{v})$. The same procedure was used in all cases.

3a Obtained as light yellow oil in $62 \%$ yield. IR (Nujol): $1654 \mathrm{~cm}^{-1} \mathrm{C}=\mathrm{N}$ (str), $2228 \mathrm{~cm}^{-1} \mathrm{CN}$ (str). ${ }^{1} \mathrm{H}$ NMR $\left(\mathrm{CDCl}_{3}\right): \delta 3.84\left(\mathrm{~s}, 3 \mathrm{H}, \mathrm{OCH}_{3}\right), 5.29\left(\mathrm{~d}, 1 \mathrm{H}, J=7.2 \mathrm{~Hz}, \mathrm{C}_{4}-\mathrm{H}\right), 5.71\left(\mathrm{~d}, 1 \mathrm{H}, J=8.4 \mathrm{~Hz}, \mathrm{C}_{5}-\mathrm{H}\right), 6.90-6.93(\mathrm{dd}, 2 \mathrm{H}$, $\mathrm{Ar}-\mathrm{H}), 6.93-6.95(\mathrm{dd}, 2 \mathrm{H}, \mathrm{Ar}-\mathrm{H}), 7.38-7.40(\mathrm{dd}, 2 \mathrm{H}, \mathrm{Ar}-\mathrm{H}), 7.78-7.80(\mathrm{dd}, 2 \mathrm{H}, \mathrm{Ar}-\mathrm{H}) .{ }^{13} \mathrm{C}$ NMR $\left(\mathrm{CDCl}_{3}\right): \delta 21.2$ (1C, 4-ㄷ), 55.1 (1C, $\left.\mathrm{OCH}_{3}\right), 66.5$ (1C, 5-C ), 114.1-114.5 (2C, Ar-C), 115.6-115.9 (2C, Ar-C), $116.2(1 \mathrm{C}, \underline{\mathrm{CN}})$, 128.4-128.7 (2C, Ar-C $), 129.2-129.5$ (2C, Ar-C), 130.3 (1C, Ar- $\underline{C}$ ), 130.6 (1C, Ar-C), 149.6 (1C, Ar(1C, 3-C), 161.7 (1C, Ar-C). MS (relative abundance) m/z: 297( $\left.\mathrm{MH}^{+}, 100\right), 270$ (32), 238 (20), 174 (16), 157 (44). Anal. Cacld. for $\mathrm{C}_{17} \mathrm{H}_{13} \mathrm{FN}_{2} \mathrm{O}_{2}, \mathrm{C}, 68.91, \mathrm{H}, 4.42$, N, 9.45\%; Found: C, 68.85, H, 4.46, N, 9.37\%.

4.2 Synthesis of 3-(4-Chlorophenyl)-5-(4-methoxyphenyl)-4,5-dihydroisoxazole-4-carbonitrile 3b: Obtained from 4-methoxy cinnamonitrile $\mathbf{1}(100 \mathrm{mg}, 0.63 \mathrm{mmol}, 1.0$ equiv) and 4-chlorobenzaldehyde oxime 2 (118mg, $0.76 \mathrm{mmol}, 1.2$ equiv), as a light yellow oil in $54 \%$ yield. IR (Nujol): $1650 \mathrm{~cm}^{-1} \mathrm{C}=\mathrm{N}(\mathrm{str}), 2220 \mathrm{~cm}^{-1} \mathrm{CN}$ (str). ${ }^{1} \mathrm{H}$ $\operatorname{NMR}\left(\mathrm{CDCl}_{3}\right): \delta 3.84\left(\mathrm{~s}, 3 \mathrm{H}, \mathrm{OCH}_{3}\right), 5.28\left(\mathrm{~d}, 1 \mathrm{H}, J=9.0 \mathrm{~Hz}, \mathrm{C}_{4}-\mathrm{H}\right), 5.70\left(\mathrm{~d}, 1 \mathrm{H}, J=9.6 \mathrm{~Hz}, \mathrm{C}_{5}-\mathrm{H}\right), 6.91-6.92(\mathrm{dd}$, $2 \mathrm{H}, \mathrm{Ar}-\mathrm{H}), 6.93-6.94(\mathrm{dd}, 2 \mathrm{H}, \mathrm{Ar}-\mathrm{H}), 7.38-7.40(\mathrm{dd}, 2 \mathrm{H}, \mathrm{Ar}-\mathrm{H}), 7.78-7.79(\mathrm{dd}, 2 \mathrm{H}, \mathrm{Ar}-\mathrm{H}) .{ }^{13} \mathrm{C}$ NMR $\left(\mathrm{CDCl}_{3}\right): \delta$ 
Synthesis of New 3,5-Diaryl-4,5-Dihydroisoxazole-4-Carbonitriles Via 1,3-Dipolar Cycloaddition

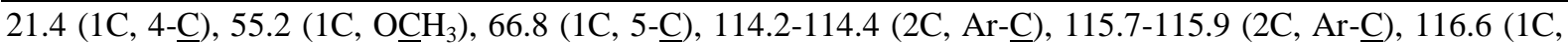

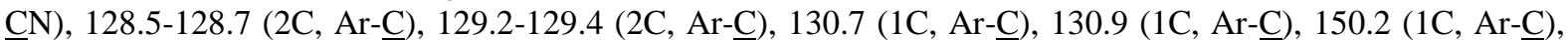
161.7 (1C, 3-ㄷ), 162.0 (1C, Ar-드). MS (relative abundance) m/z: $313\left(\mathrm{MH}^{+}, 100\right), 286$ (28), 254 (24), 174 (18), 157 (42). Anal. Cacld. for $\mathrm{C}_{17} \mathrm{H}_{13} \mathrm{ClN}_{2} \mathrm{O}_{2}, \mathrm{C}, 65.29, \mathrm{H}, 4.19, \mathrm{~N}, 8.96 \%$; Found: C, 65.19, H, 4.18, N, 8.89\%.

4.3 Synthesis of 3-(4-Bromophenyl)-5-(4-methoxyphenyl)-4,5-dihydroisoxazole-4-carbonitrile 3c: Obtained from 4-methoxy cinnamonitrile $\mathbf{1}(100 \mathrm{mg}, 0.63 \mathrm{mmol}, 1.0 \mathrm{equiv})$ and 4-bromobenzaldehyde oxime 2 (152mg, $0.76 \mathrm{mmol}, 1.2$ equiv), as a pale yellow oil in $52 \%$ yield. IR (Nujol): $1675 \mathrm{~cm}^{-1} \mathrm{C}=\mathrm{N}(\mathrm{str}), 2240 \mathrm{~cm}^{-1} \mathrm{CN}$ (str). ${ }^{1} \mathrm{H}$ $\operatorname{NMR}\left(\mathrm{CDCl}_{3}\right): \delta 3.86\left(\mathrm{~s}, 3 \mathrm{H}, \mathrm{OCH}_{3}\right), 5.19\left(\mathrm{~d}, 1 \mathrm{H}, J=7.6 \mathrm{~Hz}, \mathrm{C}_{4}-\mathrm{H}\right), 5.62\left(\mathrm{~d}, 1 \mathrm{H}, J=8.2 \mathrm{~Hz}, \mathrm{C}_{5}-\mathrm{H}\right), 6.91-6.93(\mathrm{dd}$, $2 \mathrm{H}, \mathrm{Ar}-\mathrm{H}), 6.93-6.95(\mathrm{dd}, 2 \mathrm{H}, \mathrm{Ar}-\mathrm{H}), 7.38-7.41$ (dd, $2 \mathrm{H}, \mathrm{Ar}-\mathrm{H}), 7.77-7.79(\mathrm{dd}, 2 \mathrm{H}, \mathrm{Ar}-\mathrm{H}) .{ }^{13} \mathrm{C} \mathrm{NMR}\left(\mathrm{CDCl}_{3}\right): \delta$ $22.3(1 \mathrm{C}, 4-\underline{\mathrm{C}}), 55.6\left(1 \mathrm{C}, \mathrm{OCH}_{3}\right), 66.9$ (1C, 5-C), 114.1-114.3 (2C, Ar-C), 116.8 (1C, $\left.\underline{\mathrm{CN}}\right), 125.1$ (1C, Ar-C), 126.1-126.4 (2C, Ar-C), 128.5-128.7 (2C, Ar-C), 130.1-130.3 (2C, Ar-C), 131.9 (1C, Ar-C), 133.6 (1C, Ar- ), 152.5 (1C, Ar-C), 162.5 (1C, 3-C). MS (relative abundance) $\mathrm{m} / \mathrm{z}: 357\left(\mathrm{MH}^{+},{ }^{79} \mathrm{Br} 100\right), 359\left(\mathrm{MH}^{+},{ }^{79} \mathrm{Br} 66\right), 332$ (19), 330 (28), 300 (08), 298 (25), 174 (22), 157 (46). Anal. Cacld. for $\mathrm{C}_{17} \mathrm{H}_{13} \mathrm{BrN}_{2} \mathrm{O}_{2}, \mathrm{C}, 57.16, \mathrm{H}, 3.67, \mathrm{~N}$, 7.84\%; Found: C, 57.10, H, 3.70, N, 7.78\%.

4.4 Synthesis of 3-(4-Cyanophenyl)-5-(4-methoxyphenyl)-4,5-dihydroisoxazole-4-carbonitrile 3d: Obtained from 4-methoxy cinnamonitrile $\mathbf{1}(100 \mathrm{mg}, 0.63 \mathrm{mmol}, 1.0$ equiv) and 4-cyanobenzaldehyde oxime 2 (111 $\mathrm{mg}$, $0.76 \mathrm{mmol}, 1.2$ equiv), as a colourless oil in $58 \%$ yield. IR (Nujol): $1658 \mathrm{~cm}^{-1} \mathrm{C}=\mathrm{N}(\mathrm{str}), 2234 \mathrm{~cm}^{-1} \mathrm{CN}$ (str). ${ }^{1} \mathrm{H}$ $\operatorname{NMR}\left(\mathrm{CDCl}_{3}\right): \delta 3.85\left(\mathrm{~s}, 3 \mathrm{H}, \mathrm{OCH}_{3}\right), 5.11\left(\mathrm{~d}, 1 \mathrm{H}, J=8.0 \mathrm{~Hz}, \mathrm{C}_{4}-\mathrm{H}\right), 5.54\left(\mathrm{~d}, 1 \mathrm{H}, J=8.2 \mathrm{~Hz}, \mathrm{C}_{5}-\mathrm{H}\right), 6.92-6.92(\mathrm{dd}$, $2 \mathrm{H}, \mathrm{Ar}-\mathrm{H}), 7.22-7.24$ (dd, $2 \mathrm{H}, \mathrm{Ar}-\mathrm{H}), 7.65-7.67$ (dd, $2 \mathrm{H}, \mathrm{Ar}-\mathrm{H}), 7.98-7.99$ (dd, $2 \mathrm{H}, \mathrm{Ar}-\mathrm{H}) .{ }^{13} \mathrm{C}$ NMR $\left(\mathrm{CDCl}_{3}\right): \delta$ $23.5(1 \mathrm{C}, 4-\underline{\mathrm{C}}), 55.8\left(1 \mathrm{C}, \mathrm{OCH}_{3}\right), 66.9(1 \mathrm{C}, 5-\underline{\mathrm{C}}), 114.4-114.6$ (2C, Ar- $)$ ), $115.6(1 \mathrm{C}, \mathrm{Ar}-\underline{\mathrm{C}}), 116.9(1 \mathrm{C}, \underline{\mathrm{CN}})$, 118.0 (1C, $\underline{\mathrm{CN}}$ ), 126.8-126.9 (2C, Ar- $\mathrm{C}), 129.0-129.1$ (2C, Ar-C), 131.0-131.2 (2C, Ar-C), 132.3 (1C, Ar- $\mathrm{C})$, 136.7 (1C, Ar-ㄷ), 159.7 (1C, Ar- $\underline{C}), 163.2$ (1C, 3-ㄷ). MS (relative abundance) m/z: $304\left(\mathrm{MH}^{+}, 100\right), 277$ (21), 245 (30), 174 (23), 157 (41). Anal. Cacld. for $\mathrm{C}_{18} \mathrm{H}_{13} \mathrm{~N}_{3} \mathrm{O}_{2}, \mathrm{C}, 71.28, \mathrm{H}, 4.32$, N, 13.85\%; Found: C, 71.21, H, $4.24, \mathrm{~N}, 13.79 \%$.

4.5 Synthesis of 3-(2-Chlorophenyl)-5-(4-methoxyphenyl)-4,5-dihydroisoxazole-4-carbonitrile 3e: Obtained from 4-methoxy cinnamonitrile 1 (100mg, $0.63 \mathrm{mmol}, 1.0 \mathrm{equiv})$ and 2-chlorobenzaldehyde oxime 2 (118mg, $0.76 \mathrm{mmol}, 1.2$ equiv), as a colorless oil in $55 \%$ yield. IR (Nujol): $1668 \mathrm{~cm}^{-1} \mathrm{C}=\mathrm{N}$ (str), $2235 \mathrm{~cm}^{-1} \mathrm{CN}$ (str). ${ }^{1} \mathrm{H}$ $\operatorname{NMR}\left(\mathrm{CDCl}_{3}\right): \delta 3.87\left(\mathrm{~s}, 3 \mathrm{H}, \mathrm{OCH}_{3}\right), 5.00\left(\mathrm{~d}, 1 \mathrm{H}, J=8.4 \mathrm{~Hz}, \mathrm{C}_{4}-\mathrm{H}\right), 5.50\left(\mathrm{~d}, 1 \mathrm{H}, J=8.6 \mathrm{~Hz}, \mathrm{C}_{5}-\mathrm{H}\right), 6.91-6.93(\mathrm{dd}$, $2 \mathrm{H}, \mathrm{Ar}-\mathrm{H}$ ), 7.23-7.24 (dd, 2H, Ar-H), 7.39-7.41 (dd, 2H, Ar-H), 7.669-7.681 (dd, 2H, Ar-H). Anal. Cacld. for $\mathrm{C}_{17} \mathrm{H}_{13} \mathrm{ClN}_{2} \mathrm{O}_{2}$, C, 65.29, H, 4.19, N, 8.96\%; Found: C, 65.22, H, 4.22, N, 8.89\%.

4.6 Synthesis of 3-(5-Chloro-2-nitrophenyl)-5-(4-methoxyphenyl)-4,5-dihydroisoxazole-4-carbonitrile 3f: Obtained from 4-methoxy cinnamonitrile $1(100 \mathrm{mg}, 0.63 \mathrm{mmol}, 1.0$ equiv) and 5-chloro-2-nitrobenzaldehyde oxime 2 (152mg, $0.76 \mathrm{mmol}, 1$.2equiv), as a light yellow oil in $53 \%$ yield. IR (Nujol): $1668 \mathrm{~cm}^{-1} \mathrm{C}=\mathrm{N}$ (str), 2230 $\mathrm{cm}^{-1} \mathrm{CN}(\mathrm{str}) .{ }^{1} \mathrm{H}$ NMR $\left(\mathrm{CDCl}_{3}\right): \delta 3.79\left(\mathrm{~s}, 3 \mathrm{H}, \mathrm{OCH}_{3}\right), 5.12\left(\mathrm{~d}, 1 \mathrm{H}, J=8.1 \mathrm{~Hz}, \mathrm{C}_{4}-\mathrm{H}\right), 5.51\left(\mathrm{~d}, 1 \mathrm{H}, J=9.0 \mathrm{~Hz}, \mathrm{C}_{5^{-}}\right.$ H), 6.92-6.93 (dd, 2H, Ar-H), 7.23-7.24 (dd, 2H, Ar-H), $7.70(\mathrm{dd}, 1 \mathrm{H}, \mathrm{Ar}-\mathrm{H}), 8.11(\mathrm{~d}, 1 \mathrm{H}, \mathrm{Ar}-\mathrm{H}), 8.20(\mathrm{dd}, 1 \mathrm{H}$, $\mathrm{Ar}-\mathrm{H}) .{ }^{13} \mathrm{C}$ NMR $\left(\mathrm{CDCl}_{3}\right): \delta 23.6(1 \mathrm{C}, 4-\underline{\mathrm{C}}), 55.8\left(1 \mathrm{C}, \mathrm{OCH}_{3}\right), 66.8(1 \mathrm{C}, 5-\underline{\mathrm{C}}), 114.3-114.5(2 \mathrm{C}, \mathrm{Ar}-\underline{\mathrm{C}}), 116.7$ (1C, $\underline{\mathrm{CN}}$ ), 124.1 (1C, Ar-C), 127.0-127.2 (2C, Ar-C), 130.3 (1C, Ar-C), 131.2 (1C, Ar-C), 132.8 (1C, Ar-C), 132.9 (1C, Ar-C), 136.1 (1C, Ar-C), 140.1 (1C, Ar-C), 159.9 (1C, Ar-C), 164.2 (1C, 3-C). MS (relative abundance) $\mathrm{m} / \mathrm{z}: 358\left(\mathrm{MH}^{+},{ }^{35} \mathrm{Cl}, 100\right), 360\left(\mathrm{MH}^{+},{ }^{37} \mathrm{Cl}, 33\right), 333$ (08), 331 (22), 299 (34), 174 (22), 157 (40). Anal. Cacld. for $\mathrm{C}_{17} \mathrm{H}_{12} \mathrm{ClN}_{3} \mathrm{O}_{4}, \mathrm{C}, 57.07, \mathrm{H}, 3.38, \mathrm{~N}, 11.75 \%$; Found: C, 57.00, H, 3.43, N, $11.78 \%$.

4.7 Synthesis of 3,5-Bis(4-methoxyphenyl)-4,5-dihydroisoxazole-4-carbonitrile 3g: Obtained from 4-methoxy cinnamonitrile 1 (100mg, $0.63 \mathrm{mmol}$, 1.0equiv) and 4-methoxybenzaldehyde oxime 2 (115mg, 0.76mmol, 1.2equiv), as a colourless oil in $60 \%$ yield. IR (Nujol): $1655 \mathrm{~cm}^{-1} \mathrm{C}=\mathrm{N}$ (str), $2225 \mathrm{~cm}^{-1} \mathrm{CN}$ (str). ${ }^{1} \mathrm{H}$ NMR $\left(\mathrm{CDCl}_{3}\right): \delta 3.80\left(\mathrm{~s}, 3 \mathrm{H}, \mathrm{OCH}_{3}\right), 3.81\left(\mathrm{~s}, 3 \mathrm{H}, \mathrm{OCH}_{3}\right), 5.13\left(\mathrm{~d}, 1 \mathrm{H}, J=8.1 \mathrm{~Hz}, \mathrm{C}_{4}-\mathrm{H}\right), 5.51\left(\mathrm{~d}, 1 \mathrm{H}, J=8.8 \mathrm{~Hz}, \mathrm{C}_{5}-\mathrm{H}\right)$, 6.92-6.93 (dd, 2H, Ar-H), 6.99-6.70 (dd, 2H, Ar-H), 7.22-7.23 (dd, 2H, Ar-H), 7.74-7.75 (dd, $2 \mathrm{H}, \mathrm{Ar}-\mathrm{H}) .{ }^{13} \mathrm{C}$ NMR $\left(\mathrm{CDCl}_{3}\right): \delta 23.5(1 \mathrm{C}, 4-\underline{\mathrm{C}}), 55.7\left(1 \mathrm{C}, \mathrm{OCH}_{3}\right), 55.8\left(1 \mathrm{C}, \mathrm{OCH}_{3}\right), 66.8$ (1C, 5-C), $114.3-114.4(2 \mathrm{C}, \mathrm{Ar}-\mathrm{C})$, $116.6(1 \mathrm{C}, \underline{\mathrm{CN}}), 124.2(1 \mathrm{C}, \mathrm{Ar}-\underline{-}), 127.1-127.2$ (2C, Ar- $\underline{\mathrm{C}}), 130.2(1 \mathrm{C}, \mathrm{Ar}-\underline{\mathrm{C}}), 131.2(1 \mathrm{C}, \mathrm{Ar}-\underline{\mathrm{C}}), 132.9(1 \mathrm{C}, \mathrm{Ar}-$

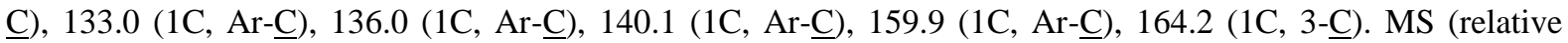
abundance) m/z: $309\left(\mathrm{MH}^{+}, 100\right), 282$ (36), 250 (32), 174 (27), 157 (42). Anal. Cacld. for $\mathrm{C}_{18} \mathrm{H}_{16} \mathrm{~N}_{2} \mathrm{O}_{3}, \mathrm{C}, 70.12$, H, 5.23, N, 9.09\%; Found: C, 70.06, H, 5.26, N, 9.10\%.

4.8 Synthesis of 3-(3,4-Dimethoxyphenyl)-5-(4-methoxyphenyl)-4,5-dihydroisoxazole-4-carbonitrile 3h: Obtained from 4-methoxy cinnamonitrile $\mathbf{1}(100 \mathrm{mg}, 0.63 \mathrm{mmol}$, 1.0equiv) and 3,4-dimethoxybenzaldehyde oxime 2 (138mg, $0.76 \mathrm{mmol}, 1.2$ equiv), as a colourless oil in $61 \%$ yield. IR (Nujol): $1672 \mathrm{~cm}^{-1} \mathrm{C}=\mathrm{N}$ (str), 2238 $\mathrm{cm}^{-1} \mathrm{CN}(\mathrm{str}) .{ }^{1} \mathrm{H}$ NMR $\left(\mathrm{CDCl}_{3}\right): \delta 3.81\left(\mathrm{~s}, 6 \mathrm{H}, \mathrm{OCH}_{3}\right), 3.81\left(\mathrm{~s}, 3 \mathrm{H}, \mathrm{OCH}_{3}\right), 5.12\left(\mathrm{~d}, 1 \mathrm{H}, J=8.5 \mathrm{~Hz}, \mathrm{C}_{4}-\mathrm{H}\right), 5.52$ (d, $\left.1 \mathrm{H}, J=9.2 \mathrm{~Hz}, \mathrm{C}_{5}-\mathrm{H}\right), 6.92-6.93(\mathrm{dd}, 2 \mathrm{H}, \mathrm{Ar}-\mathrm{H}), 6.99$ (dd, $\left.1 \mathrm{H}, \mathrm{Ar}-\mathrm{H}\right), 7.23-7.24(\mathrm{dd}, 2 \mathrm{H}, \mathrm{Ar}-\mathrm{H}), 7.30(\mathrm{~s}, 1 \mathrm{H}$, $\mathrm{Ar}-\mathrm{H}), 7.40(\mathrm{dd}, 1 \mathrm{H}, \mathrm{Ar}-\mathrm{H}) .{ }^{13} \mathrm{C} \mathrm{NMR}\left(\mathrm{CDCl}_{3}\right): \delta 23.4(1 \mathrm{C}, 4-\underline{\mathrm{C}}), 55.8\left(1 \mathrm{C}, \mathrm{OCH}_{3}\right), 55.8\left(1 \mathrm{C}, \mathrm{OCH}_{3}\right), 55.8(1 \mathrm{C}$, 
Synthesis of New 3,5-Diaryl-4,5-Dihydroisoxazole-4-Carbonitriles Via 1,3-Dipolar Cycloaddition

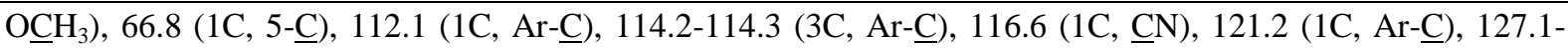

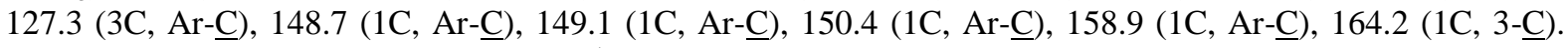
MS (relative abundance) m/z: $339\left(\mathrm{MH}^{+}, 100\right), 312$ (19), 280 (33), 174 (26), 157 (45). Anal. Cacld. for $\mathrm{C}_{19} \mathrm{H}_{18} \mathrm{~N}_{2} \mathrm{O}_{4}, \mathrm{C}, 67.44, \mathrm{H}, 5.36, \mathrm{~N}, 8.28 \%$; Found: C, 67.38, H, 5.32, N, 8.21\%.

4.9 Synthesis of 5-(4-Methoxyphenyl)-3-phenyl-4,5-dihydroisoxazole-4-carbonitrile 3i: Obtained from 4methoxy cinnamonitrile 1 (100mg, $0.63 \mathrm{mmol}, 1.0 \mathrm{equiv})$ and benzaldehyde oxime 2 (92mg, $0.76 \mathrm{mmol}$, 1.2equiv), as a colourless oil in $56 \%$ yield. IR (Nujol): $1670 \mathrm{~cm}^{-1} \mathrm{C}=\mathrm{N}$ (str), $2235 \mathrm{~cm}^{-1} \mathrm{CN}$ (str). ${ }^{1} \mathrm{H}$ NMR $\left(\mathrm{CDCl}_{3}\right): \delta 3.82\left(\mathrm{~s}, 3 \mathrm{H}, \mathrm{OCH}_{3}\right), 5.13\left(\mathrm{~d}, 1 \mathrm{H}, J=9.0 \mathrm{~Hz}, \mathrm{C}_{4}-\mathrm{H}\right), 5.53\left(\mathrm{~d}, 1 \mathrm{H}, J=8.5 \mathrm{~Hz}, \mathrm{C}_{5}-\mathrm{H}\right), 6.91-6.93(\mathrm{dd}, 2 \mathrm{H}$, $\mathrm{Ar}-\mathrm{H}), 7.20-7.22(\mathrm{dd}, 2 \mathrm{H}, \mathrm{Ar}-\mathrm{H}), 7.56-7.78(\mathrm{~m}, 5 \mathrm{H}, \mathrm{Ar}-\mathrm{H}) .{ }^{13} \mathrm{C}$ NMR $\left(\mathrm{CDCl}_{3}\right): \delta 23.4(1 \mathrm{C}, 4-\underline{\mathrm{C}}), 55.8(1 \mathrm{C}$, $\left.\mathrm{OCH}_{3}\right), 66.7(1 \mathrm{C}, 5-\mathrm{C}), 114.3(2 \mathrm{C}, \mathrm{Ar}-\mathrm{C}), 116.6(1 \mathrm{C}, \mathrm{CN}), 127.0$ (2C, Ar-C), $128.2(2 \mathrm{C}, \mathrm{Ar}-\mathrm{C}), 128.4(2 \mathrm{C}, \mathrm{Ar}-$ C), 131.1 (1C, Ar-C), 133.0 (1C, Ar-C), 133.6 (1C, Ar-C), 159.0 (1C, Ar-C), 164.7 (1C, 3-C). MS (relative abundance) m/z: $279\left(\mathrm{MH}^{+}, 100\right), 252$ (22), 220 (30), 174 (20), 157 (38). Anal. Cacld. for $\mathrm{C}_{17} \mathrm{H}_{14} \mathrm{~N}_{2} \mathrm{O}_{2}, \mathrm{C}, 73.37$, H, 5.07, N, 10.07\%; Found: C, 73.30, H, 5.01, N, 10.02\%.

\section{Conclusion}

The successful synthesis of new compounds with the use of $\mathrm{CrO}_{2}$ as catalytic dehydrogenating agents validates the significance of this study. However, the modification in the procedure for improving the yield of the products remains of interest.

\section{Acknowledgements}

One of the authors (PJR) is grateful to the University Grants Commission, New Delhi, for the award of Teacher Fellowship and Financial Support.

\section{References:}

[1] Rolf Huisgen, "1,3-Dipolar cycloadditions. Past and future", Angewandte Chemie Int Ed. Engl., 2, 1963, 565-632.

[2] P. Caramella, P.Grunanger in "1,3-Dipolar Cycloaddition Chemistry", ed. A. Padwa, John Wiley and Sons, New York. Vol 1, 1984, 337-345.

[3] Pinho E Melo, M.V.D. Teresa "Recent advances on the synthesis and reactivity of isoxazoles", Curr. Org. Chem., 9(10), 2005, 925958.

[4] Patrizia Cali, Lars Naerum, Seema Mukhija, Anders Hjelmencrantz, "Isoxazole-3-hydroxamic acid derivatives as peptide deformylase inhibitors and potential antibacterial agents", Bioorg. and Med. Chem. Letters, 14(24), 2004, 5997-6000.

[5] K. Ajay Kumar, M. Govindaraju, G. Vasantha Kumar, "Synthesis of isoxazoles via 1,3-dipolar cycloaddition reactions and their antimicrobial activity", Indian J. of Heterocyclic Chem, 20, 2010, 183-84.

[6] E. Abdu Musad, R. Mohamed, B.A. Saeed, B.S. Vishwanath, K.M.L. Rai, "Synthesis and evaluation of antioxidant and antibacterial activities of new substituted bis(1,3,4-oxadiazoles), 3,5-bis(substituted) pyrazoles and isoxazoles", Bioorg. and Med. Chem. Letters, 21(12), 2011, 3536-3540.

[7] Michael Rowley, Howard B. Broughton, Ian Collins, Raymond Baker, Frances Emms, Rosemarie Marwood, Shil Patel, Smita Patel, C.Ian Ragan, Stephen B. Freedman, Paul D. Leeson, "5-(Chlorophenyl)-4-methyl-3-(1-(2-phenylethyl)piperidin-4-yl)isoxazole: A potent, selective antagonist at human cloned dopamine D4 receptors", J. Med. Chem., 39, 1996, 1943-1945.

[8] John J. Talley, David L. Brown, Jeffery S. Carter, Matthew J. Graneto, Carol M. Koboldt, Jaime L. Masferrer, William E. Perkins, Roland S. Rogers, Alexander F. Shaffer, Yan Y. Zhang, Ben S. Zweifel, Karen Seibert, "4-[5-Methyl-3-phenylisoxazol-4-yl]benzenesulfonamide, valdecoxib: A potent and selective inhibitor of COX-2", J. Med. Chem., 43, 2000, 775-777.

[9] Maria Paola Giovannoni, Claudia Vergelli, Carla Ghelardini, Nicoletta Galeotti, Alessandro Bartolini, and Vittorio Dal Piaz, "[3Chlorophenyl)piperazinypropyl]pyridazinones and analogues as potent antinociceptive agents", J. Med. Chem., 46, 2003, 1055-1059.

[10] W.-T. Li, D.-R. Hwang, C.-P. Chen, C.-W.Shen, C.-L. Huang, T.-W. Chen, C.-H. Lin, Y.-L. Chang, Y.-Y. Chang, Y.-K. Lo, H.-Y. Tseng, C.-C. Lin, J.-S. Song, H.-C. Chen, S.-J. Chen, S.-H. Wu, C.-T. Chen, "Synthesis and biological evaluation of $N$-heterocyclic indolyl glyoxylamides as orally active anticancer agents", J. Med. Chem., 46, 2003, 1706-1715.

[11] John W. Patterson, Paul S. Cheung, Michael J. Ernest, "3-Carboxy-5-methyl-N-[4-(trifluoromethyl)phenyl]-4-isoxazolecaboxamide, new prodrug for the antiarthritic agent 2-cyano-3-hydroxy-N-[4-(trifluoromethyl)phenyl]-2-butenamide", J. Med. Chem., 35, 1992, 507-510.

[12] Vijay V. Dabholkar, Faisal Y. Ansari, "Synthesis and characterization of selected fused isoxazole and pyrazole derivatives and their antimicrobial activity", J. Serb. Chem. Soc., 74(11), 2009, 1219-1228.

[13] V.V. Kachhadia, M.R. Patel, H.S. Joshi, "Synthesis of isoxazoles and cyanopyridines bearing benzo $(b)$ thiophene nucleus as potential antitubercular and antimicrobial agents", Journal of Sciences, Islamic Republic of Iran, 15(1), 2004, 47-51.

[14] A.P. Kozikowski, "The isoxazoline route to the molecules of nature", Acc. Chem. Res., 17(12), 1984, 410-416.

[15] K.M. Lokanatha Rai, N. Linganna, Alfred Hassner, C. Anjanamurthy, "A convenient method for the generation of nitrile oxide and its application to the synthesis of 2-isoxazolines", Org. Prep. Proc. Int., 24, 1992, 91-93.

[16] Sandeep Bhosale, Dnyaneshwar P. Sonune, Uppuleti Viplava Prasad, Debnath Bhuniya, "Inverse kinetic isotope effect in Magtrieve mediated oxidation or deoximination of benzaldoxime: mechanistic implication", Tetrahedron Letters, 53(14), 2012, $1794-1797$.

[17] Hui Wan, Yanqing Peng, "Clean Synthesis of Azo Compounds Using Magtrieve in the Ionic Liquid [bmim][Br]", Monatsh Chem. Prep. Org. Chem., 139, 2008, 909-912.

[18] K. Ajay Kumar, K.M. Lokanatha Rai, K.B. Umesha, "Synthesis and evaluation of antifungal and antibacterial activity of ethyl 3,5diarylisoxazole-4-carboxylates", J. Chem. Res (S), 2001, 436-438.

[19] K. Ajay Kumar, K.M. Lokanatha Rai, K.B. Umesha, K. Rajasekhara Prasad, "Synthesis of 3-aryl-5N-aryl-4,6-dioxo-pyrrolo[3,4-d]7,8-dihydroisoxazoles", Indian J. Chem., 40B, 2001, 269-273.

[20] K.B. Umesha, K.M. Lokanatha Rai, K. Ajay Kumar, "A novel synthesis of isoxazoles via 1,3-dipoar cycloaddition of nitrile oxides to acetyl acetone", Synth. Commun., 32(12), 2002, 1841-1846. 